\title{
Comparative analysis of proso millet (Panicum miliaceum L.) leaf transcriptomes for insight into drought tolerance mechanisms
}

\author{
Yuyu Zhang ${ }^{\dagger}$, Xiaoli Gao ${ }^{\dagger}$, Jing Li, Xiangwei Gong, Pu Yang, Jinfeng Gao, Pengke Wang and Baili Feng*
}

\begin{abstract}
Background: Drought stress is a major abiotic stress that causes huge losses in agricultural production. Proso millet (Panicum miliaceum L.) can efficiently adapt to drought stress and provides important information and gene resources to improve drought tolerance. However, its complex drought-responsive mechanisms remain unclear.

Results: Among 37 core Chinese proso millet cultivars, Jinshu 6 (JS6) was selected as the drought-sensitive test material, whereas Neimi 5 (NM5) was selected as the drought-tolerant test material under PEG-induced water stress. After sequencing, 1695 differentially expressed genes (DEGs) were observed in JS6 and NM5 without PEG-induced water stress (JS6CK and NM5CK). A total of 833 and 2166 DEGs were found in the two cultivars under simulated drought by using 20\% PEG-6000 for 6 (JS6T6 and NM5T6) and $24 \mathrm{~h}$ (JS6T24 and NM5T24), respectively. The DEGs in JS6T6 and JS6T24 treatments were approximately 0.298- and 0.754-fold higher than those in NM5T6 and NM5T24, respectively. Compared with the respective controls, more DEGs were found in T6 treatments than in T24 treatments. A delay in the transcriptional responses of the ROS scavenging system to simulated drought treatment and relatively easy recovery of the expression of photosynthesis-associated genes were observed in NM5. Compared with JS6, different regulation strategies were observed in the jasmonic acid (JA) signal transduction pathway of NM5.
\end{abstract}

Conclusion: Under PEG-induced water stress, NM5 maintained highly stable gene expression levels. Compared with drought-sensitive cultivars, the different regulation strategies in the JA signal transduction pathway in droughttolerant cultivars may be one of the driving forces underlying drought stress tolerance.

Keywords: Drought, Proso millet, RNA-seq, Gene expression, Jasmonic acid

\section{Background}

Drought stress is a chronic, random, and unpredictable abiotic stress that causes huge losses in agricultural crop production [1-3]. Drought was considered a major cause of severe food shortages and famine [2]. Present statistical data have revealed that $40 \%$ of the world's agricultural production comes from irrigated lands, which accounts for $70 \%$ of the world's water reservoir [4]. It remains a major limitation of agricultural production. With the

\footnotetext{
* Correspondence: fengbaili@nwsuaf.edu.cn

'Yuyu Zhang and Xiaoli Gao contributed equally to this work.

College of Agronomy, Northwest A\&F University / State Key Laboratory of

Crop Stress Biology in Arid Areas, Yangling 712100, Shaanxi, China
}

increase in world population and global warming, drought stress will exacerbate in the future due to limited water resources [5-7]. Therefore, to improve agricultural production, drought tolerance mechanisms must be understood.

With satisfactory adaptation to drought, high temperature, and poor soil conditions, proso millet (Panicum miliaceum L.) is important in tasks related to drought resistance [8-10]. It is a $\mathrm{C}_{4}$ crop and tetraploid species $(2 N=4 \mathrm{X}=36)$ with a short growing season [11]. Proso millet is one of the oldest cultivated cereals with great historical significance [12, 13] and is mainly planted as a source of staple food and fodder in the semi-arid regions of China $[14,15]$. It is also planted as

(c) The Author(s). 2019 Open Access This article is distributed under the terms of the Creative Commons Attribution 4.0 International License (http://creativecommons.org/licenses/by/4.0/), which permits unrestricted use, distribution, and reproduction in any medium, provided you give appropriate credit to the original author(s) and the source, provide a link to the Creative Commons license, and indicate if changes were made. The Creative Commons Public Domain Dedication waiver (http://creativecommons.org/publicdomain/zero/1.0/) applies to the data made available in this article, unless otherwise stated. 
a makeup crop in case of major crop failure [15]. Therefore, insights into the drought tolerance mechanisms and the important genetic resources of proso millet will help improve its drought tolerance and that of other crops.

Drought-responsive mechanisms, including various molecular, morphological, and physiological responses, help plants survive drought stress and maintain productivity. Plants have morphological and physiological responses, including improvement of root traits [16], reduction of epidermal (stomatal and cuticular) conductance and decrease in evaporative surface (leaf area) and radiation absorption [3], to cope with water-deficit conditions. Hormones regulate the complex network of signal transduction to adjust and control plant growth in stress-free situations and survive under stress [3]. When plants experience stress, the first step involves perception of environmental signals [17]. In the presence of abiotic stresses, abscisic acid (ABA), an important plant hormonal signal, provides one of the fastest responses and triggers subsequent defences [18]. It is responsible for regulating stomatal closure [19], which is the primary way of saving water via gaseous exchange in plant leaves. In response to abiotic stress, the complex cross talk among different plant hormones causes synergetic or antagonistic effects [20]. ABA and jasmonic acid (JA) have played positive roles in regulating stomatal closure, whereas auxins and cytokinins have played negative roles $[3,21]$. Moreover, the role of ethylene is determined by different tissues under varying conditions [3].

JA plays important roles in stress response by regulating the balance of plant growth and defence response and enabling plants to adapt to changing conditions [22-24]. Water stress induces JA accumulation to promote the synthesis of ascorbate peroxidase, monodehydroascorbate reductase, glutathione reductase, dehydroascorbate reductase, ascorbic acid, and glutathione [25]. The ascorbate-glutathione cycle in plant cells highly contributes to scavenging reactive oxygen species (ROS) [26]. This cycle works in all the organelles where ROS detoxification is needed [27, 28]. According to Bartoli et al. [29], the abundance of ascorbate is responsible for the regulation of cellular redox buffering capacity, which influences the threshold of activating hormone signals and the interactions between different hormones. In addition, apoplastic ascorbate helps alleviate the enhanced oxidative burden from stresses and is the first line of defence against potentially damaging external oxidants [30].

Under normal conditions, ROS are inevitable products of aerobic metabolic processes [31-33], including photosynthesis and respiration; ROS levels are usually low and can be strictly controlled [26]. However, water stress can disrupt cellular homeostasis and increase ROS production, thereby triggering specific oxidative responses [26]. Under stress, ROS are secondary messengers in signalling cascades that convey important information to regulate hormonal changes in concentrations and/or sensitivity to activate downstream stress responses and defence processes [29]. However, ROS are overgenerated during drought stress [31-33] and can oxidize key cellular components and cause oxidative cell destruction [28, 34]. Moreover, overgeneration of ROS is fatal to protein, membrane, and DNA and can cause cell death [35-37], thereby posing a major threat to plant cells. Superoxide dismutase (SOD), catalase (CAT), ascorbate, glutathione, and peroxidase (POD) are also involved in various defence responses $[2,38]$ and play key roles in ROS scavenging under biotic and abiotic stresses [39].

Under drought conditions, genes regulate the pathways related to osmoprotectants to directly protect important proteins and membranes [40]. Adverse conditions can induce the responses of membrane transporters and ion channels related to water and ion uptake [41]. These adverse conditions can induce related transcription factors that regulate the downstream stress-related genes to cope with water stress [2]. Knowledge of genomic information is crucial to understand the molecular mechanism of drought tolerance and provide important gene resources. Recently, RNA sequencing (RNA-seq), which is widely used in various plants, has proven to be a highly efficient method to achieve transcriptome data [42-44]. However, previous studies on drought tolerance of proso millet mainly focused on its morphological and physiological responses [45]. In recent years, several studies [13, 45] have reported the transcriptome characteristics of proso millet. However, the mechanism underlying leaf response and adaptation to drought through changes at the molecular level remains unclear, particularly among different drought-tolerant cultivars.

In the present study, to further elucidate the molecular basis of the response of proso millet leaves to drought stress and the drought tolerance mechanisms of different drought-tolerant cultivars, we selected drought-sensitive and drought-tolerant cultivars. Their transcriptome was analyzed based on RNA-seq data. Our results will provide information that can help improve our understanding of the molecular mechanism underlying drought tolerance. Moreover, this study provides important gene resources for improving the tolerance of proso millet and other crops.

\section{Results}

Evaluation of the drought tolerance of proso millet cultivars Among the 37 cultivars, the mean germination rate of the control group was approximately $92.3 \%$, and their highest and lowest germination rates were 99.0 and $74.0 \%$, respectively. The mean germination rate of the 
simulated drought treatment group was $72.9 \%$, and their highest and lowest germination rates 92.3 and 43.0\%, respectively. The mean relative germination rate was $79.0 \%$, and the highest and lowest relative germination rates were 101.6 and $46.6 \%$, respectively (Additional file 1: Table S2). The germination rates of the control group exceeded 95\%, and the highest and lowest relative germination rates of the simulated drought treatment group were 96.9 and 48.6\%, respectively. Among these cultivars, Neimi 5 and Jinshu 6 (renamed NM5 and JS6, respectively) were selected as drought-tolerant and drought-sensitive test materials for subsequent analysis, respectively.

\section{Differences in malondialdehyde (MDA) contents in NM5} and JS6 under PEG-induced water stress

In the control group, JS6 and NM5 were designated as JS6CK and NM5CK, respectively. In the group of $20 \%$ PEG-6000 treatment for $6 \mathrm{~h}$, JS6 and NM5 were designated as JS6T6 and NM5T6, respectively. In the group of 20\% PEG-6000 treatment for 24 h, JS6 and NM5 were designated as JS6T24 and NM5T24, respectively. MDA contents were elevated in both cultivars as the stress time continued (Fig. 1). JS6 consistently showed higher MDA contents than NM5, regardless of the presence or absence of PEG-induced water stress. Specifically, the MDA contents in JS6 were approximately 0.584-, 1.042-, and 0.706-fold greater than those in NM5 under normal conditions, T6 treatment, and T24 treatment, respectively. Compared with the MDA content under normal conditions, the MDA contents in the JS6 cultivar increased by 95.6 and $152.9 \%$ under T6 and T24 treatments, respectively. Compared with the control, the MDA contents in the NM5 cultivar increased by 51.8 and $134.8 \%$ under T6 and T24 treatments, respectively.

\section{RNA sequencing and de novo assembly}

Samples for RNA-seq were obtained from 18 libraries of the two cultivars (containing three biological replicates). All the raw reads of RNA sequencing data were deposited in the NCBI Short Read Archive database under the accession number SRP144636 (SAMN08947059SAMN08947076), as associated with the BioProject PRJNA454008. About 42 million high-quality reads were obtained from each sample (Table 1). A total of 115,660 transcripts and 59,035 unigenes were obtained with a mean length of $1367 \mathrm{bp}$ and N50 of $2044 \mathrm{bp}$ and a mean length of $1080 \mathrm{bp}$ and N50 of $1953 \mathrm{bp}$, respectively. The transcriptome shotgun assembly data were deposited at DDBJ/EMBL/GenBank under accession number GHHA01000000.

\section{Functional annotation}

A total of 33,634 unigenes were annotated in at least one database among the 59,035 unigenes, including 32, 410 unigenes annotated in the $\mathrm{nr}$ database, 22,328 unigenes in SWISS-PROT, 9988 unigenes in KEGG, and 18,124 unigenes in the COG database (Fig. 2).

KEGG pathway enrichment analysis revealed that 5631 unigenes were annotated in known pathways. The genes related to endocytosis were the most abundant in the cellular process class, and the genes involved in plant hormone signal transduction accounted for the largest part of the environmental information processing class.

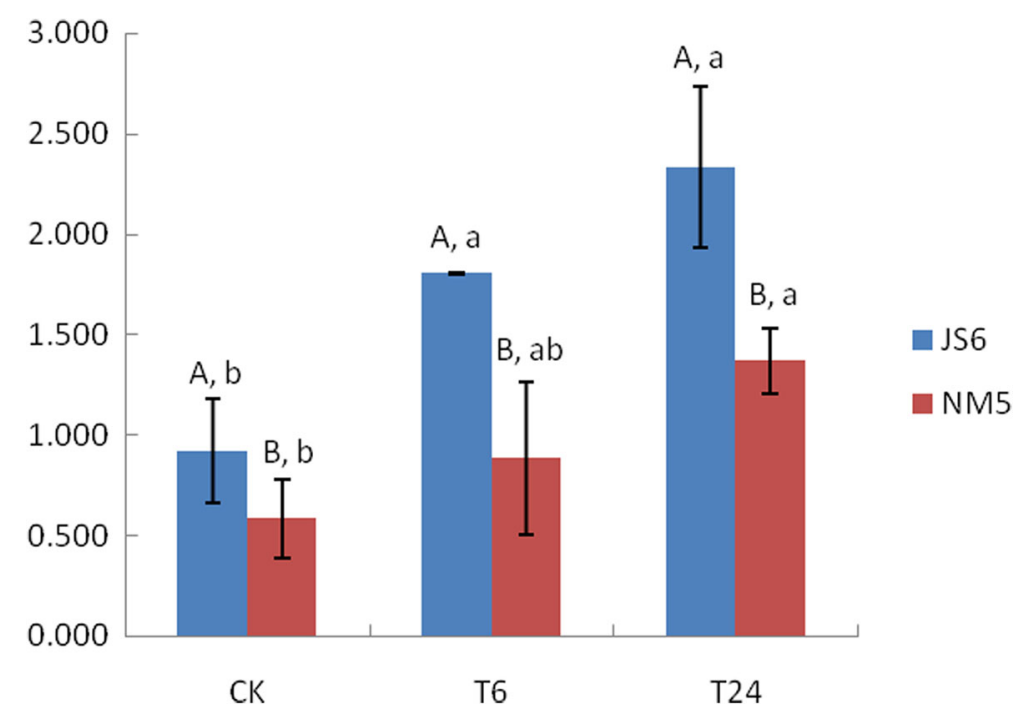

Fig. 1 MDA content changes in the leaves of JS6 and NM5. JS6 and NM5 plants at the three-leaf stage were treated with 20\% PEG-6000 solution for $6 \mathrm{~h}$ (JS6T6 and NM5T6, respectively), $24 \mathrm{~h}$ (JS6T24 and NM5T24, respectively), or kept in water (control). After treatments, leaf tissues from each group were sampled for MDA measurement. All data are shown as mean \pm standard error. Different uppercase letters represent significant differences between cultivars; different lowercase letters indicate significant differences between treatments (Duncan test; $P<0.05$ ) 
Table 1 Information of RNA-seq performed for proso millet leaf tissue in the presence or absence of simulated drought treatment

\begin{tabular}{|c|c|c|c|c|}
\hline Sample & Total bases & Total reads & Quality filtered bases (\%) & Quality filtered reads (\%) \\
\hline JS6CK-1 & $6,487,530,000$ & $43,250,200$ & $6,314,524,327(97.33 \%)$ & $42,131,554(97.41 \%)$ \\
\hline JS6CK-2 & $6,509,234,400$ & $43,394,896$ & 6,363,855,996(97.77\%) & $42,458,184(97.84 \%)$ \\
\hline JS6CK-3 & $6,696,348,000$ & $44,642,320$ & 6,557,437,381(97.93\%) & $43,748,814(98.00 \%)$ \\
\hline NM5CK-1 & $6,247,170,600$ & $41,647,804$ & $6,107,549,061(97.77 \%)$ & 40,747,270(97.84\%) \\
\hline NM5CK-2 & $6,747,776,700$ & $44,985,178$ & $6,571,843,868(97.39 \%)$ & $43,846,186(97.47 \%)$ \\
\hline NM5CK-3 & $5,807,890,200$ & $38,719,268$ & $5,688,608,498(97.95 \%)$ & $37,951,394(98.02 \%)$ \\
\hline JS6T6-1 & $6,057,733,500$ & $40,384,890$ & $5,943,426,904(98.11 \%)$ & $39,651,746(98.18 \%)$ \\
\hline JS6T6-2 & $7,144,457,400$ & $47,629,716$ & 6,993,164,558(97.88\%) & $46,654,198(97.95 \%)$ \\
\hline JS6T6-3 & $7,505,556,300$ & $50,037,042$ & $7,331,461,113(97.68 \%)$ & $48,914,060(97.76 \%)$ \\
\hline NM5T6-1 & $6,195,031,200$ & $41,300,208$ & $6,073,088,662(98.03 \%)$ & $40,518,262(98.11 \%)$ \\
\hline NM5T6-2 & $5,989,206,900$ & $39,928,046$ & $5,878,371,054(98.15 \%)$ & $39,216,888(98.22 \%)$ \\
\hline NM5T6-3 & $6,496,346,700$ & $43,308,978$ & 6,323,637,814(97.34\%) & $42,190,100(97.42 \%)$ \\
\hline JS6T24-1 & $5,748,725,700$ & $38,324,838$ & $5,646,195,618(98.22 \%)$ & $37,667,488(98.28 \%)$ \\
\hline JS6T24-2 & $7,100,685,000$ & $47,337,900$ & 6,930,777,593(97.61\%) & $46,242,434(97.69 \%)$ \\
\hline JS6T24-3 & $6,987,597,600$ & $46,583,984$ & 6,831,757,689(97.77\%) & 45,579,628(97.84\%) \\
\hline NM5T24-1 & $6,640,053,600$ & $44,267,024$ & $6,523,588,317(98.25 \%)$ & $43,521,448(98.32 \%)$ \\
\hline NM5T24-2 & $5,929,631,400$ & $39,530,876$ & $5,824,098,355(98.22 \%)$ & $38,854,876(98.29 \%)$ \\
\hline NM5T24-3 & $5,753,017,800$ & $38,353,452$ & $5,656,189,224(98.32 \%)$ & $37,733,478(98.38 \%)$ \\
\hline Average & $6,446,888,500$ & $42,979,257$ & $6,308,865,335$ & $42,090,445$ \\
\hline
\end{tabular}

Genes related to ribosome, spliceosome, protein processing in the endoplasmic reticulum, and RNA transport were highly abundant in the genetic information processing class. Those involved in carbon metabolism and biosynthesis of amino acids accounted for the largest part of the metabolism class, and those related to plant-pathogen interaction were the most abundant in the organismal system class (Additional file 1: Table S3).

In accordance with gene ontology (GO) term analysis, 10,085 unigenes were annotated in three major GO classes, namely, 5892 unigenes in cellular component, 6676 unigenes in molecular function, and 6680 unigenes in

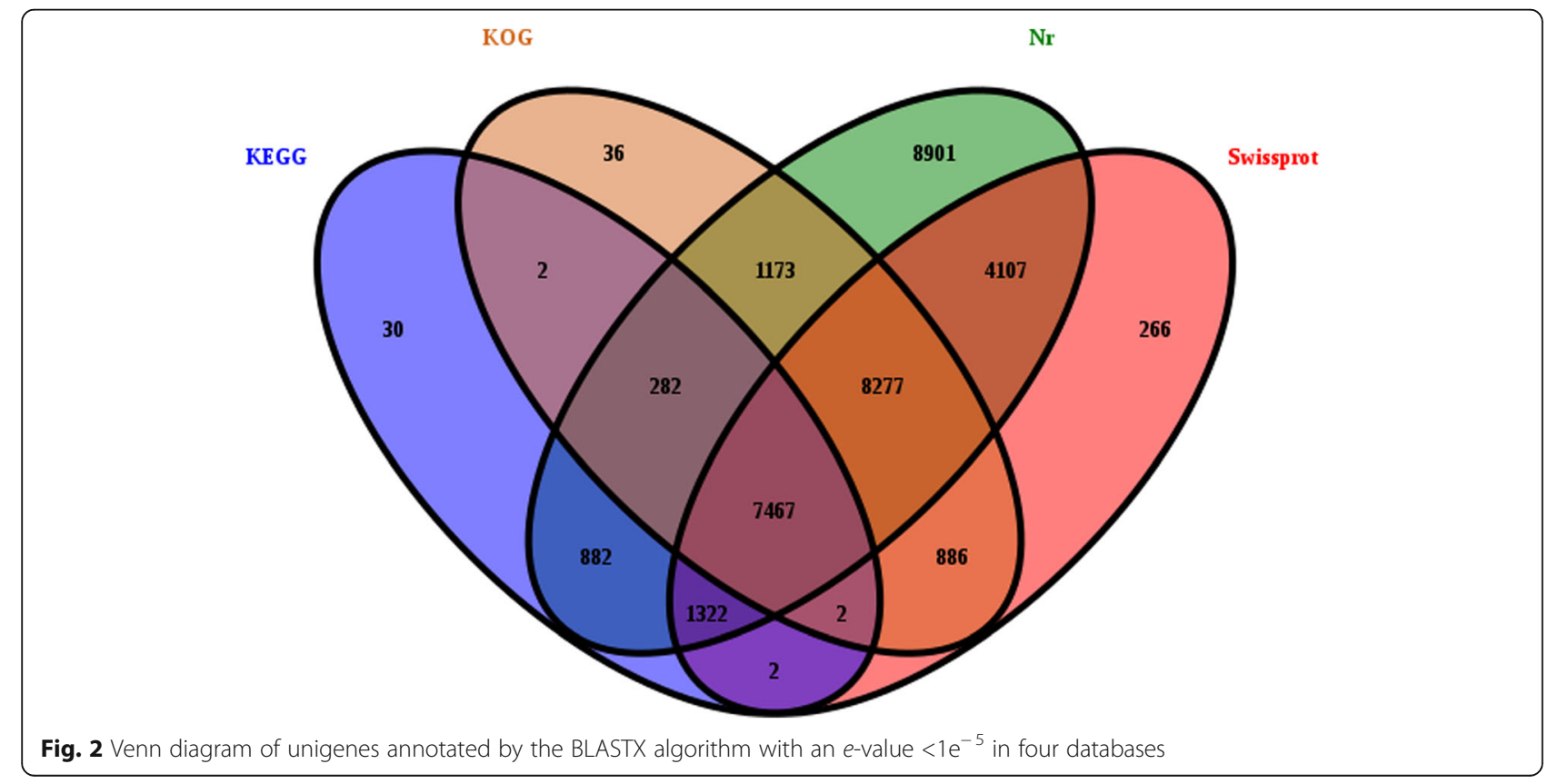


biological process. The unigenes related to "membrane", "membrane part", "cell", and "cell part" were highly abundant in the cellular component class. In terms of biological process, the unigenes were highly abundant in "metabolic process" and "cellular process" categories, while the genes involved in "catalytic activity" and "binding" accounted for the largest and second largest parts of molecular function class, respectively (Fig. 3).

\section{Overall analysis of differentially expressed genes (DEGs) in JS6 and NM5 cultivars}

In the control group, 1695 DEGs were observed in JS6 and NM5. Among the samples collected after simulated drought treatment for 6 and 24h, 833 and 2166 DEGs were identified in JS6 and NM5, respectively (Fig. 4a). The Venn diagram showed that 207 genes were differentially expressed in the two cultivars in the control and simulated drought treatment groups (Fig. 4b).

Compared with their respective control groups, 11,375 DEGs were identified in JS6T6 (including 6553 upregulated and 4822 down-regulated genes), 8762 DEGs were found in NM5T6 (including 4702 up-regulated and 4060 down-regulated genes), 4406 DEGs were noted in JS6T24 (including 2588 up-regulated and 1818 downregulated genes), and 2512 DEGs were observed in NM5T24 (including 1069 up-regulated and 1443 downregulated genes; Fig. 4a). Numerous DEGs were found in the T6 groups in both cultivars. DEGs in JS6T6 and JS6T24 treatments were approximately 0.298 - and 0.754- fold higher than those in NM5T6 and NM5T24, respectively. Comparisons of GO categories and KEGG pathways between the cultivars showed that the DEGs in both cultivars were involved in similar GO categories and KEGG pathways (Additional file 1: Tables S4 and S5). On the basis of the Euler diagram, the comparisons of the control and T6 groups revealed that 1) 2334 genes (1207 up- and 1127 down-regulated genes) were specifically regulated in NM5, whereas 4947 genes (3071 up- and 1876 downregulated genes), which were 1.12 -fold more than that in NM5, were specifically regulated in JS6; 2) compared with their respective control groups, 3468 and 2919 genes were co-up- or co-down-regulated in both cultivars, respectively, whereas 14 and 27 genes were up- and downregulated in JS6T6 but down- and up-regulated in NM5T6, respectively (Fig. 4c). The comparisons of the control and T24 groups revealed that 1) 1344 DEGs (558 up- and 786 down-regulated) were specifically regulated in NM5, whereas 3238 DEGs (1994 up- and 1244 downregulated), which were 1.41-fold higher than those in NM5, were specifically regulated in JS6; 2) 488 and 551 genes were co-up- or co-down-regulated in both cultivars, respectively, whereas 106 and 23 genes were up- and down-regulated in JS6T24 but down- and up-regulated in NM5T24, respectively (Fig. 4d).

\section{DEGs involved in oxidative stress responses}

Compared with their respective controls, more genes belonging to POD, SOD, and APX families were induced

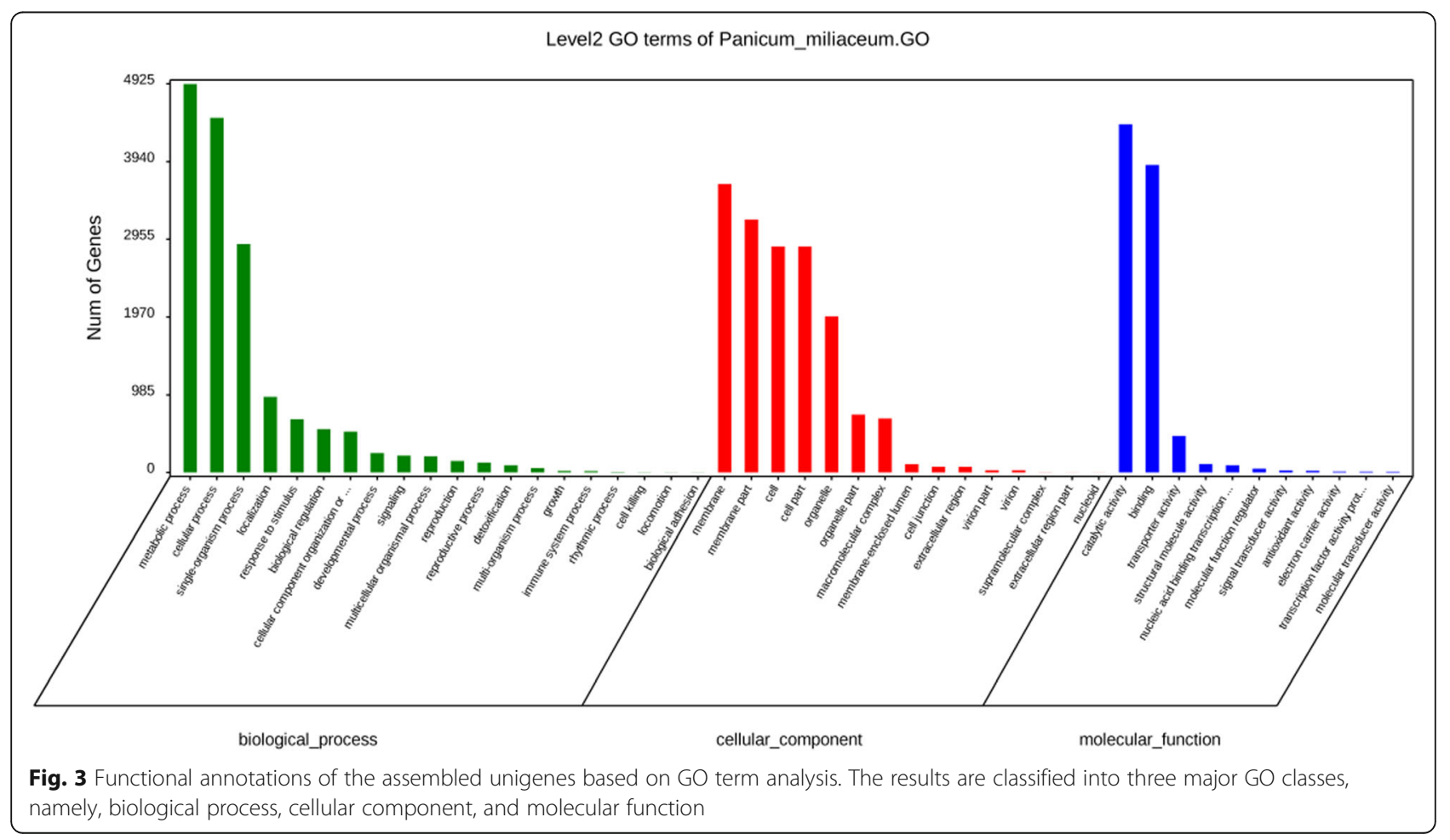



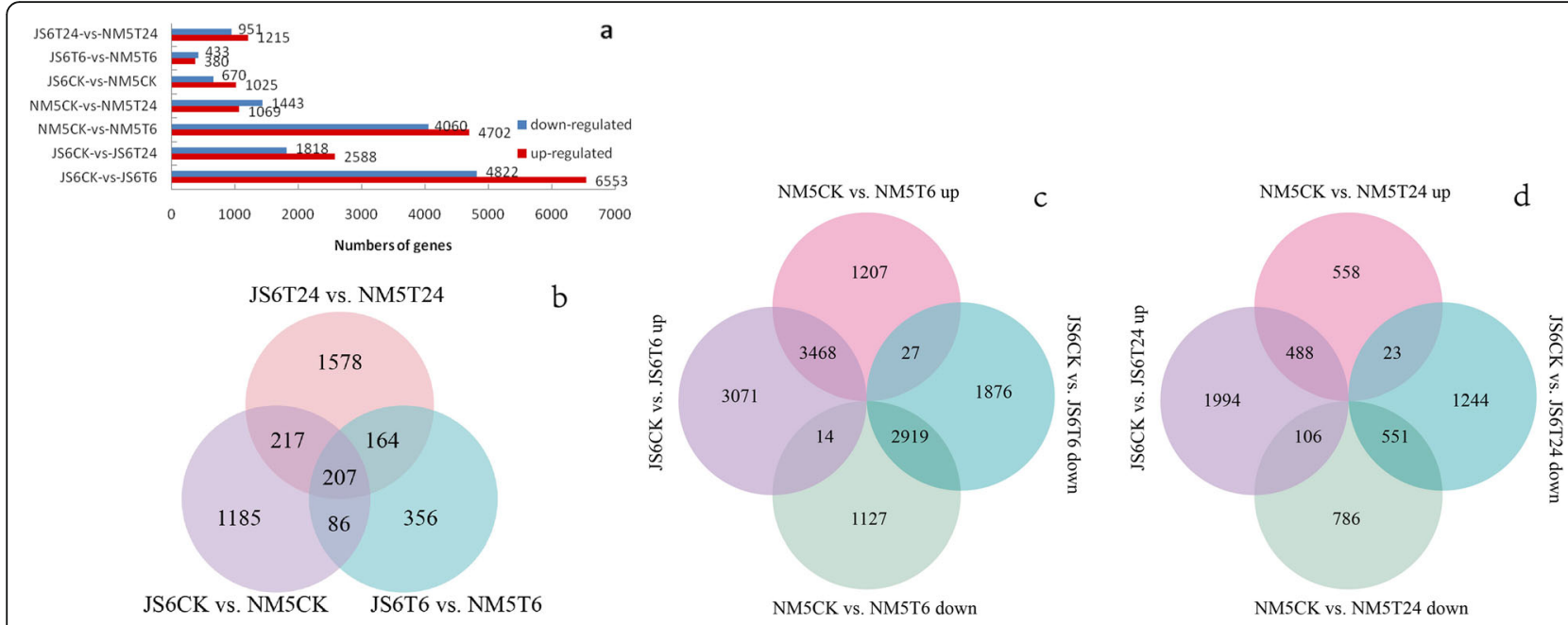

Fig. 4 Differentially expressed genes (DEGs) in the presence and absence of simulated drought. DEGs were selected based on a cut-off of FDR $\leq$ 0.05 and $|\log 2 \mathrm{FC}| \geq 1$. a Summary of the number of DEGs in the presence and absence of simulated drought. $\mathbf{b}$ Venn diagram indicating the DEGs from comparisons of JS6CK versus NM5CK, JS6T6 versus NM5T6, and JS6T24 versus NM5T24. c Euler diagram of DEGs from comparisons between the control group and T6 treatment, including up- and down-regulated genes in JS6T6 and NM5T6. d Euler diagram of DEGs from comparisons between the control group and T24 treatment, including up- and down-regulated genes in JS6T24 and NM5T24

in JS6 than in NM5 under T6 treatment, whereas more genes belonging to POD, SOD, and CAT families were induced in NM5 than in JS6 after simulated drought treatment from $6 \mathrm{~h}$ to $24 \mathrm{~h}$ (Fig. 5a). Specifically, regardless of the presence or absence of PEG-induced water stress, more genes belonging to POD and APX families exhibited higher expression levels in NM5 than in JS6. After simulated drought treatment, more genes belonging to SOD families showed higher expression levels in NM5 than in JS6 (Fig. 5b). Interestingly, more than 57$70 \%$ of the genes belonging to the APX family and $75 \%$ of genes belonging to the CAT family from both cultivars were up-regulated, whereas few genes belonging to the POD and SOD families from both cultivars were induced after PEG-induced water stress for $6 \mathrm{~h}$. However, after PEG-induced water stress from $6 \mathrm{~h}$ to $24 \mathrm{~h}$, an increase in the up-regulated genes belonging to the POD and SOD families and decrease in the up-regulated genes belonging to the APX and CAT families were observed (Fig. 5a).

\section{DEGs involved in osmotic stress responses}

Regardless of the presence or absence of PEG-induced water stress, more genes related to proline biosynthesis maintained higher expression levels in NM5 than in JS6 (Fig. 6). However, compared with their respective controls, more genes involved in proline biosynthesis were up-regulated in JS6 than in NM5 under simulated drought treatment (Fig. 6).

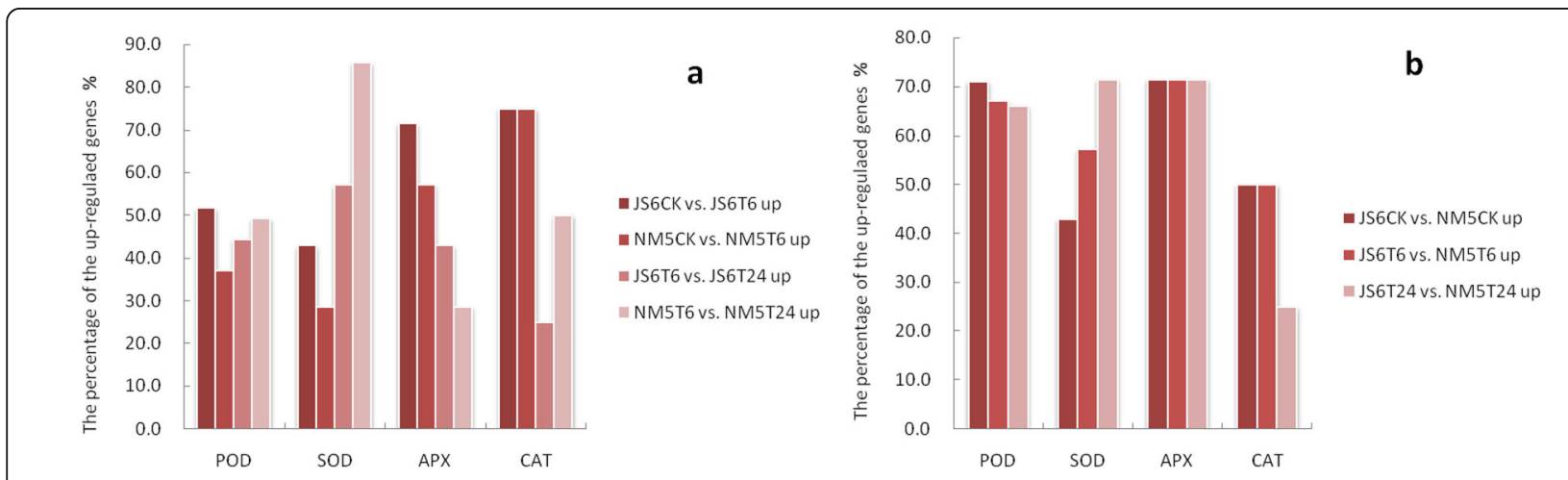

Fig. 5 ROS scavenging enzyme-related genes in JS6 and NM5 differentially expressed in the presence and absence of simulated drought. DEGs were selected based on a cut-off of FDR $\leq 0.05$ and $|\log 2 \mathrm{FC}| \geq 1$. a Percentages of these up-regulated DEGs from comparisons of JS6CK versus JS6T6, NM5CK versus NM5T6, JS6T6 versus JS6T24, and NM5T6 versus NM5T24. b Percentages of these up-regulated DEGs from comparisons of JS6CK versus NM5CK, JS6T6 versus NM5T6, and JS6T24 versus NM5T24 


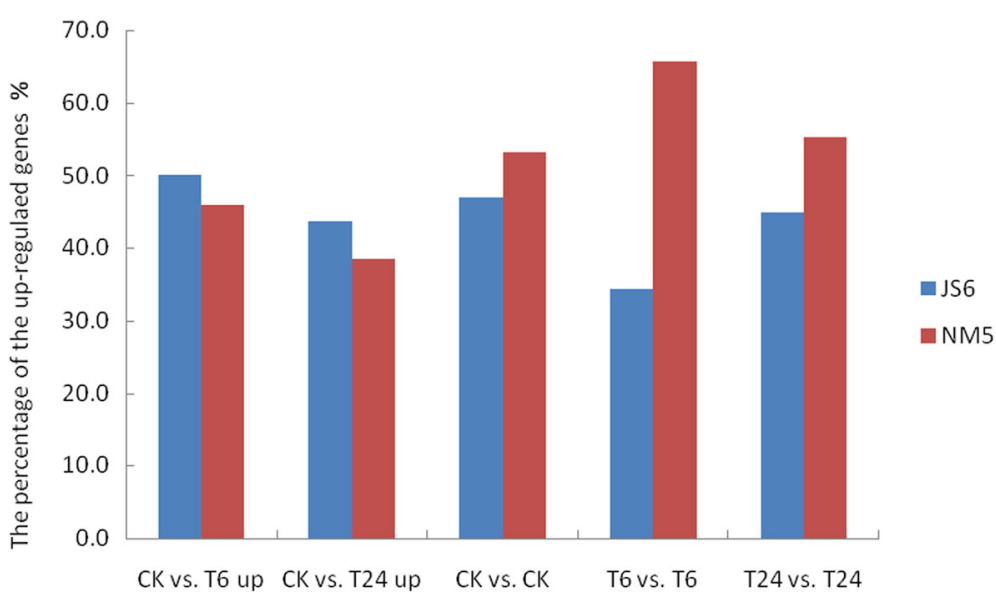

Fig. 6 Proline-related genes in JS6 and NM5 differentially expressed in the presence and absence of simulated drought. DEGs were selected based on a cut-off of FDR $\leq 0.05$ and $|\log 2 \mathrm{FC}| \geq 1$. Blocks from left to right indicate the percentages of these up-regulated DEGs from comparisons of JS6CK versus JS6T6, NM5CK versus NM5T6, JS6CK versus JS6T24, NM5CK versus NM5T24, JS6CK versus NM5CK, JS6T6 and NM5T6, and JS6T24 versus NM5T24

\section{DEGs involved in photosynthesis-associated responses}

The photosynthesis-related genes were mostly suppressed under T6 and returned to being up-regulated after PEG-induced water stress from $6 \mathrm{~h}$ to $24 \mathrm{~h}$ in both cultivars (Fig. 7a). Compared with their respective controls, these genes were most repressed under T24 in JS6 but most induced under T24 in NM5. Compared with their respective control groups, 26 genes were co-downregulated in both cultivars under T6 treatment, whereas four genes were down-regulated in JS6T6 but upregulated in NM5T6. 25 genes were co-up-regulated after simulated drought treatment from $6 \mathrm{~h}$ to $24 \mathrm{~h}$ in

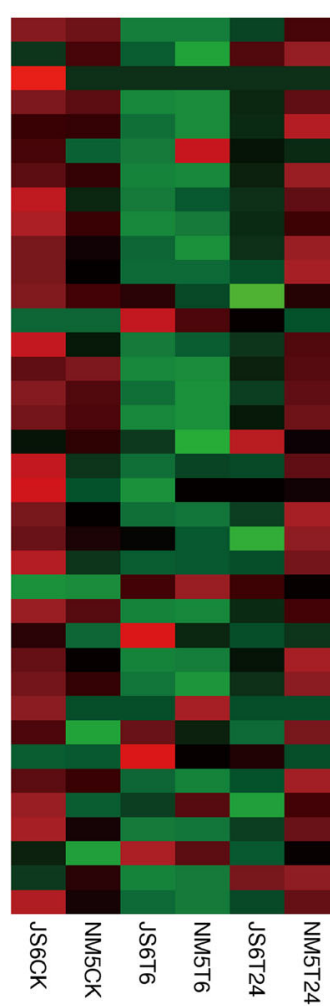

Unigene000666 Unigene000669 Unigene001610 Unigene002552 Unigene 002552 Unigene002654 Unigene002744 Unigene 002788 Unigene 005416 Unigene 008291 Unigene009025 Unigene009026 Unigene010216 Unigene013943 Unigene014159 Unigene 015560 Unigene016721 Unigene016787 Unigene018752 Unigene01969 Unigene019695 Unigene 025556 Unigene025556 Unigene027329
Unigene030872 Unigene 030872 Unigene041744 Unigene 047567 Unigene 04925 Unige 04925 Unigene 049253 Unigene04925 Unigene 049256 Unigene049257 Unigene 049452 Unigene049817 Unigene051513 Unigene 052576 Unigene052576 Unigene053852

2
0
-1
-2

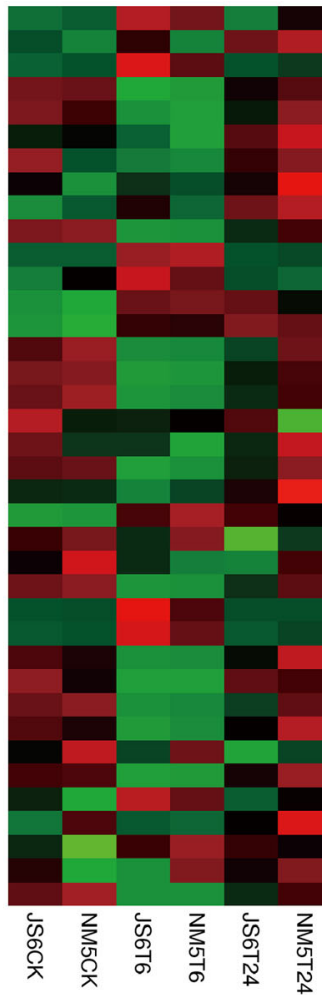

Unigene 003135 Unigene 004106 Unigene 004211 Unigene008304 Unigene 011455 Unigene 015488 Unigene 015489 Unigene 015490 Unigene 015491 Unigene015544 Unigene 018081 Unigene 018720 Unigene 018721 Unigene 018722 Unigene023196 Unigene 023197 Unigene023198 Unigene023198 Unigene025592 Unigene 025986 Unigene 03305 Unigene 034325 Unigene 034928 Unigene 036898 Unigene 036898 Unigene 038857 Unigene 039748 Unigene 039749 Unigene 040775 Unigene 04374 Unigene 043791 Unigene 044962 Unigene 045576 Unigene 049786

Unigene 052576

Unigene 052576

Unigene 053029

Unigene 055344 Unigene 056380

Fig. 7 Comparisons of photosynthesis- (a) and chlorophyll-related genes (b) in JS6 and NM5 in the presence and absence of simulated drought. The heat map indicates the relative transcript levels of the genes 
both cultivars. 25 genes were down-regulated in JS6T24 but up-regulated in NM5T24 compared with their respective controls (Additional file 2: Figure S1a-c). Similar expression profiles were observed in genes related to chlorophyll content (Fig. 7b). Compared with their respective controls, 20 genes were co-downregulated in both cultivars under T6 treatment, whereas four genes were down-regulated in JS6T6 but upregulated in NM5T6. 23 genes were co-up-regulated after drought treatment from $6 \mathrm{~h}$ to $24 \mathrm{~h}$ in both cultivars. 14 genes were co-up-regulated in both cultivars under T24 treatment, whereas 11 genes were down-regulated in JS6T24 but up-regulated in NM5T24 compared with their respective controls (Additional file 2: Figure S2a-c).

\section{Expression patterns of the DEGs}

All these DEGs from each cultivar at different treatments were clustered separately in eight profiles (Fig. 8). In accordance with the profiles, considerable differences were observed in gene expression with stress time in response to PEG-induced water stress between the two cultivars. The DEGs were significantly enriched in profiles 2,5 , and $6(p<0.05)$ in JS6 but significantly enriched in profiles 2 and $5(p<0.05)$ in NM5. Profiles 2 , 5, and 6 contained 4037, 3657, and 3329 DEGs in JS6, respectively, whereas profiles 2 and 5 included 4397 and 4615 DEGs in NM5, respectively.

The GO categories of "organic substance metabolic process", "cellular metabolic process", and "primary metabolic process" were enriched in the biological processes in both cultivars. "Membrane part" and "intrinsic component of membrane" were the most abundant categories in the cellular component, and genes involved in "organic cyclic compound binding" and "heterocyclic compound binding" were enriched in the molecular function of the cultivars (Additional file 1: Table S6).

KEGG pathway enrichment analysis annotated 1943 and 1738 DEGs from JS6 and NM5, respectively. Some important KEGG pathways, including the top 10 pathways with the highest quantities of DEGs and the photosynthesis pathway, are shown in Additional file 1: Table S7. These pathways included plant hormone signal transduction, carbon metabolism, ribosome, phenylpropanoid biosynthesis, biosynthesis of amino acids, starch and sucrose metabolism, protein processing in endoplasmic reticulum, plant-pathogen interaction, and spliceosome and purine metabolism, or glycolysis/gluconeogenesis.

\section{Expression patterns of the DEGs involved in abiotic and biotic stress response-related biological processes}

Genes involved in "response to stimulus" were enriched in profiles 5 and 6 in JS6, whereas these genes were enriched in profile 5 in NM5 (Fig. 9a). Similar findings were observed in the $\mathrm{GO}$ categories of "biological regulation" in both cultivars (Fig. 9a). The main expression patterns of these genes showed that they were promoted at T6 but suppressed at T24 (profile 5) or maintained high expression level at T24 (profile 6) in JS6. By contrast, the promotion of these genes at $\mathrm{T} 6$ and the repression at T24 (profile 5), which is the main expression pattern, were observed in NM5.

Compared with their respective controls, approximately $28.7 \%$ more DEGs related to "response to stimulus" were identified in JS6T6 than in NM5T6, including $55.7 \%$ more up-regulated genes. Moreover, $32.8 \%$ more DEGs related to "biological regulation" were observed in JS6T6 than in NM5T6, including 59.1\% more upregulated genes. Approximately $83.0 \%$ more DEGs

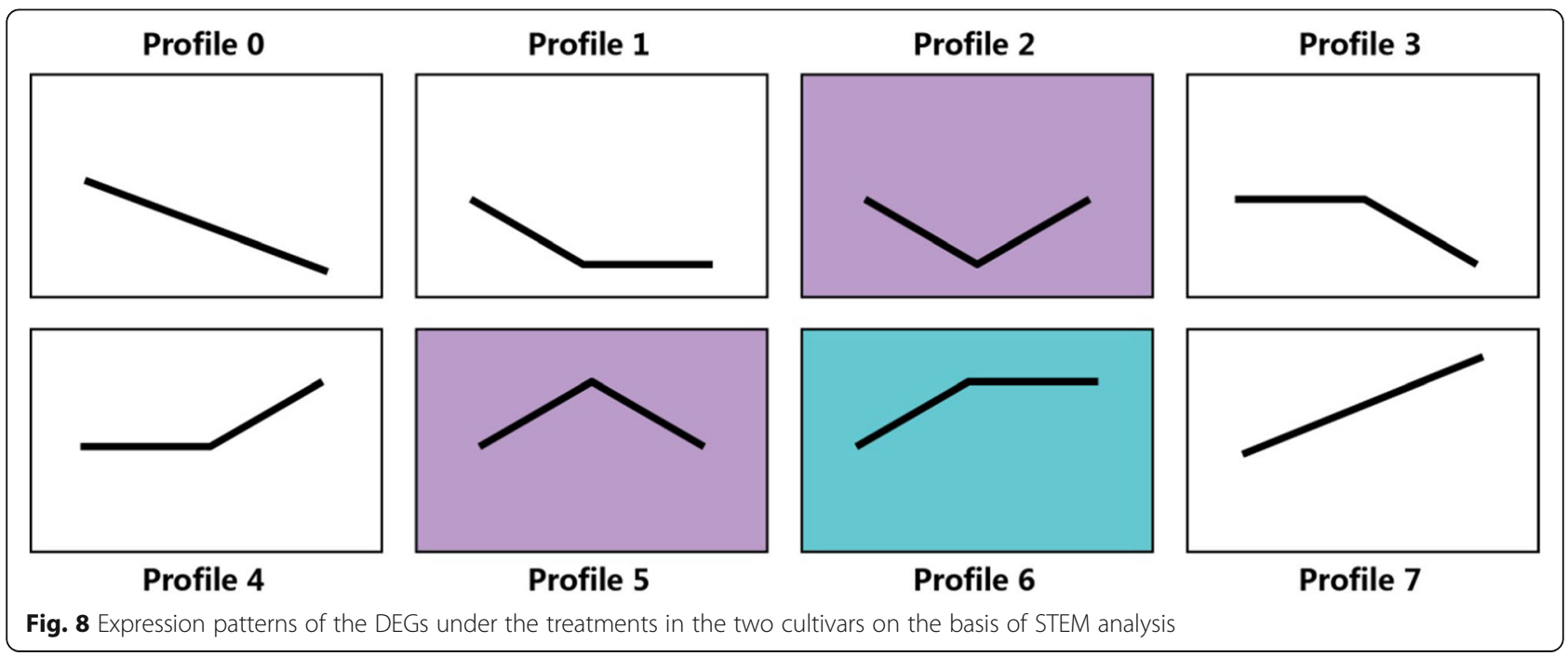




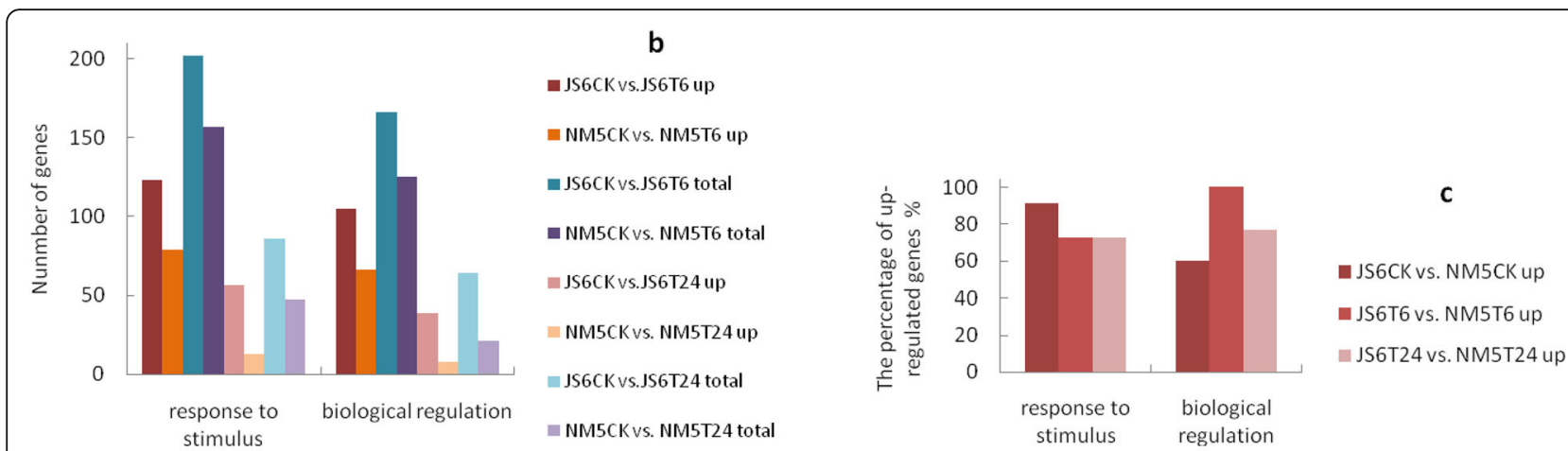

a
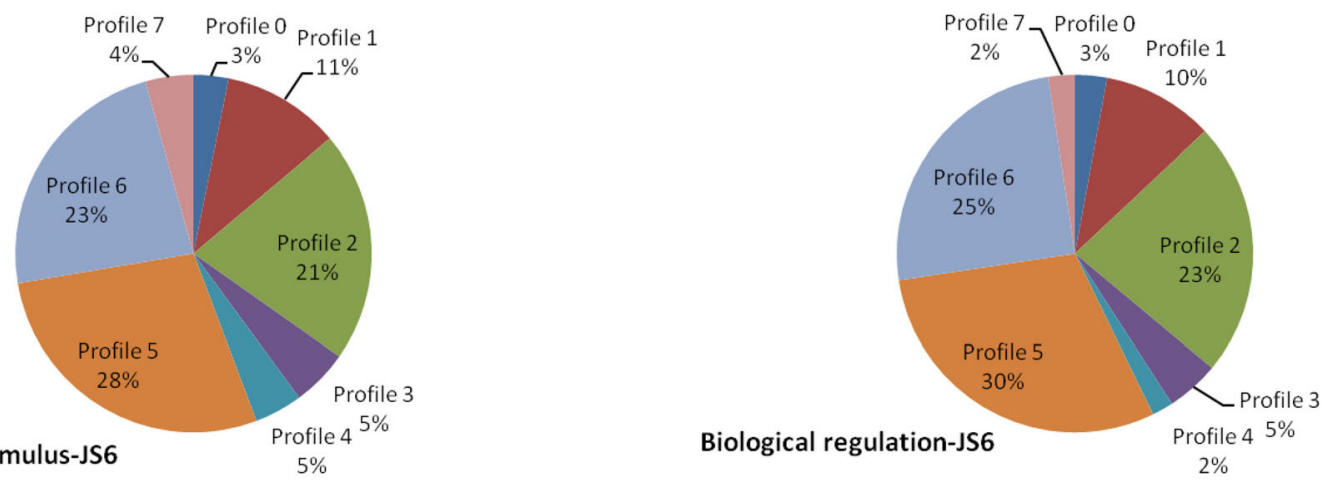

Response to stimulus-JS6
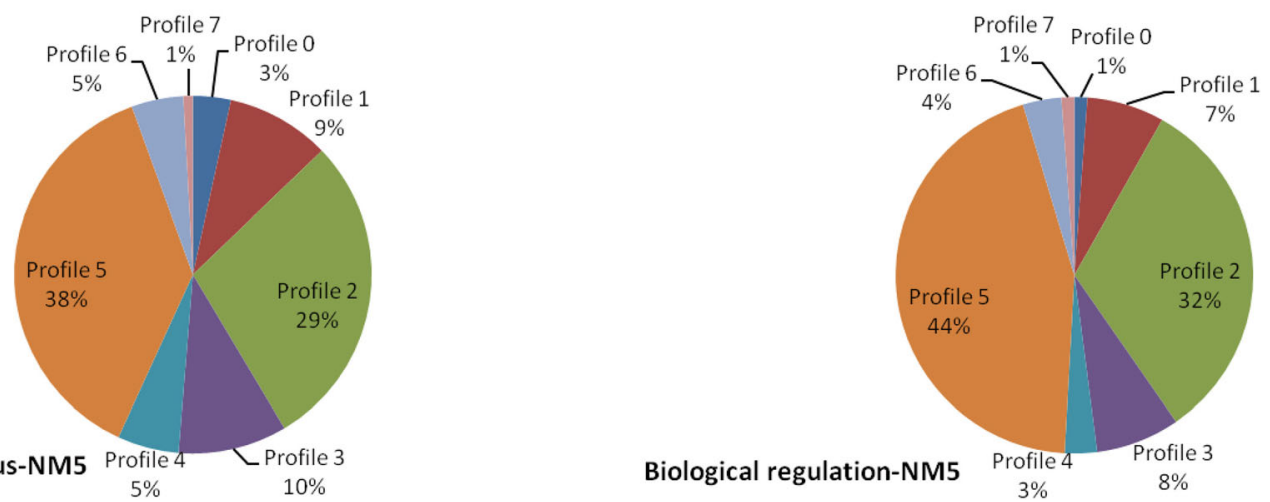

Fig. 9 Genes involved in abiotic and biotic stress response-related biological processes in JS6 and NM5 differentially expressed in the presence and absence of simulated drought. DEGs were selected based on a cut-off of FDR $\leq 0.05$ and $|\log 2 \mathrm{FC}| \geq 1$. a Expression patterns of these DEGs under the treatments in the two cultivars. $\mathbf{b}$ Comparisons of these DEGs drawn from JS6CK versus JS6T6, NM5CK versus NM5T6, JS6CK versus JS6T24, and NM5CK versus NM5T24. c Comparisons of these up-regulated DEGs drawn from JS6CK versus NM5CK, JS6T6 versus NM5T6, and JS6T24 versus NM5T24

related to "response to stimulus" were identified in JS6T24 than in NM5T24, including approximately 3.31fold more up-regulated genes. More than twice more DEGs related to "biological regulation" were identified in JS6T24 than in NM5T24, including approximately 3.88fold more up-regulated genes (Fig. 9b). However, more genes in NM5 involved in "response to stimulus" and "biological regulation" showed higher expression levels than those in JS6, regardless of the presence or absence of PEG-induced water stress (Fig. 9c).
Expression patterns of the DEGs involved in plant hormone signal transduction and photosynthesis pathways

A total of 122 and 121 DEGs were annotated to plant hormone signal transduction pathways in JS6 and NM5, respectively, and these items showed the highest representations. About 45\% (55/121) of the genes were enriched in profile 5 in NM5, which accounted for the largest part; however, 34\% (41/122) of the genes were enriched in profile 6 and 29\% (35/122) were enriched in profile 5 in JS6, accounting for the largest and second 
largest parts in JS6 (Fig. 10a), respectively. Moreover, 40 and 34 DEGs were annotated to the photosynthesis pathways in JS6 and NM5, respectively. Approximately $73 \%(25 / 34)$ of the genes were enriched in profile 2 in NM5, which accounted for the largest part, and none of the genes belonged to profile 1 in NM5. However, 48\% $(19 / 40)$ of the genes were enriched in profile 1, and 33\% $(13 / 40)$ of the genes were enriched in profile 2 in JS6, which accounted for the largest and second largest parts in JS6 (Fig. 10b), respectively.

We focused on two pairwise comparisons, namely, JS6T6 versus NM5T6 and JS6T24 versus NM5T24, in the analysis of plant hormone signal transduction pathways (Fig. 11). Compared with JS6, four unigenes encoding auxin-induced protein AUX/IAA were up-regulated under T6, four unigenes were up-regulated under T24, and two unigenes were down-regulated under T24 in NM5. A repressed unigene encoding ARF and an upregulated unigene encoding SAUR were found in NM5T6, whereas two up-regulated and one downregulated unigenes involved in SAUR encoding were identified in NM5T24. Unigene 049967 encoding AHP and Unigene 000689 encoding CRE1 were suppressed in NM5T6 and NM5T24, respectively. Unigene039784, Unigene039785, and Unigene040255 encoding A-ARR were up-regulated under T24 in NM5. Two of the unigenes involved in encoding PP2C were down-regulated under T6 in NM5, and two other unigenes were suppressed under T24 in NM5. Unigene 014459 and Unigene 058993 involved in the jasmonate ZIM domain proteins (JAZ) encoding were repressed under T6 in NM5. Moreover, Unigene 016717 and Unigene 058993 were suppressed under T24 in NM5.

\section{qRT-PCR validation}

Both qRT-PCR and RNA-seq data demonstrated that the expression levels of the eight transcripts, namely, Unigene 000666, Unigene 002552, Unigene 002654, Unigene 008291, Unigene 038857, Unigene 043791, Unigene 050227, and Unigene 056380, were repressed at T6 and promoted at T24 (Fig. 12); by contrast, Unigene 003461 and Unigene 056050 were up-regulated at T6 a

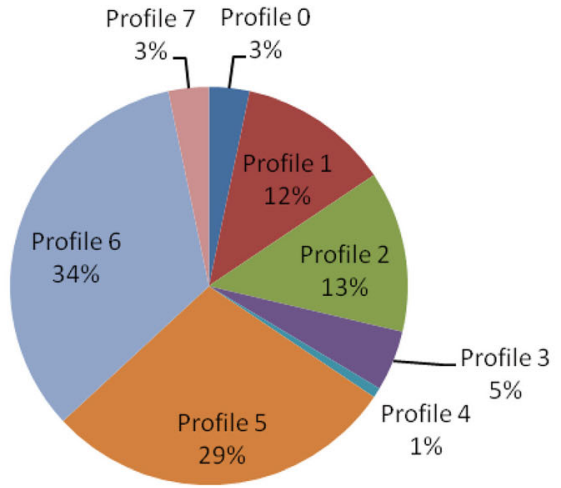

Plant hormone signal transduction-JS6

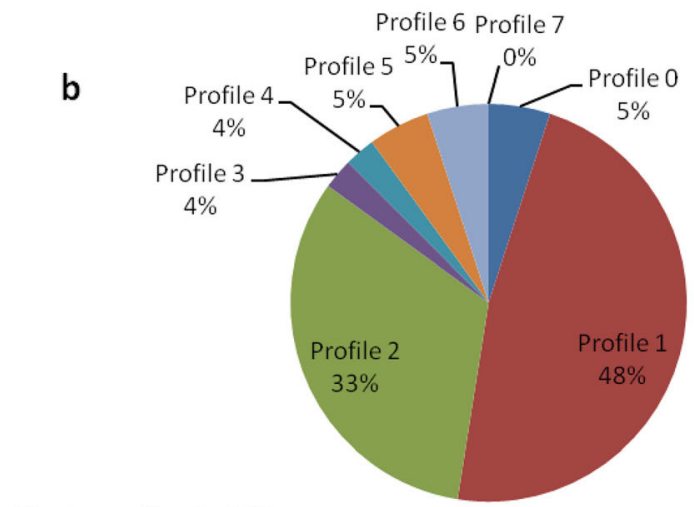

Photosynthesis-JS6

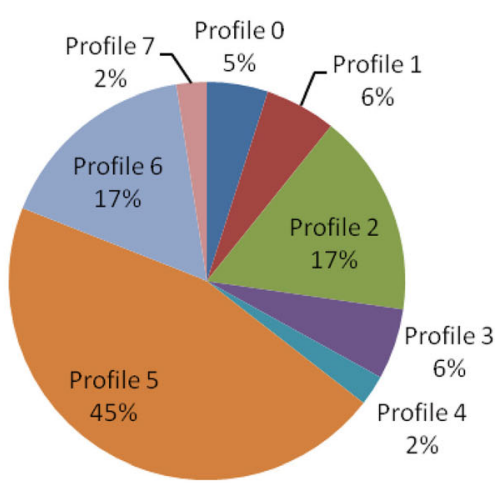

Plant hormone signal transduction-NM5

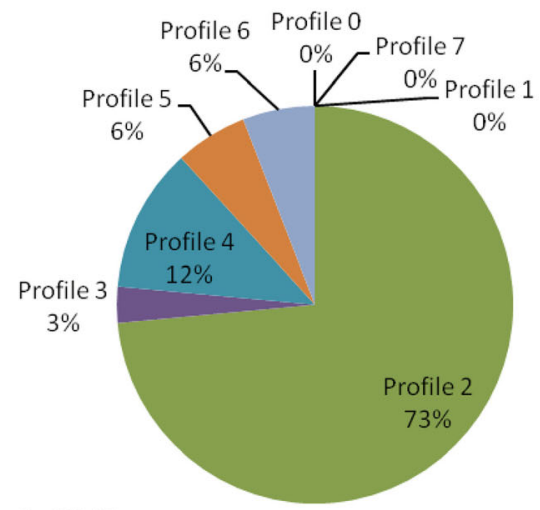

Photosynthesis-NM5

Fig. 10 Genes involved in plant hormone signal transduction and photosynthesis pathways in JS6 and NM5 differentially expressed in the presence and absence of simulated drought. DEGs were selected based on a cut-off of FDR $\leq 0.05$ and $|\log 2 \mathrm{FC}| \geq 1$. a Expression patterns of the DEGs involved in the plant hormone signal transduction pathway under the treatments in the two cultivars. $\mathbf{b}$ Expression patterns of the DEGs involved in the photosynthesis pathway under the treatments in the two cultivars 


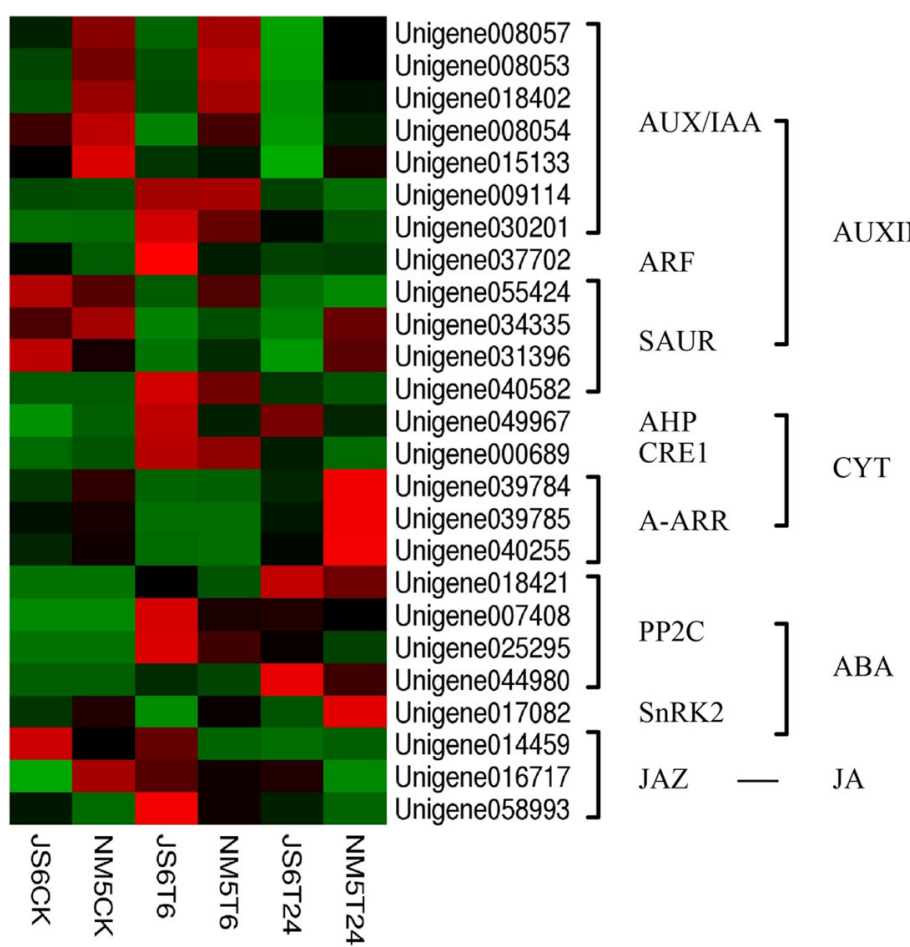

Fig. 11 Comparisons of the genes involved in the plant hormone signal transduction pathways in JS6 and NM5 in the presence and absence of simulated drought treatments. The heat map indicates the relative transcript levels of the genes

but down-regulated at T24. The expression patterns of these transcripts obtained by qRT-PCR were highly consistent with the RNA-seq results (Figs. 12 and 13). A significantly positive correlation between the RNA-seq and qRT-PCR data was revealed via linear regression analysis (Fig. 13), thereby confirming the DEGs data from RNA-seq.

\section{Discussion}

Diverse cultivars with different genetic backgrounds provide important genetic resources to breed new varieties with improved traits [46]. Proso millet is highly adaptive to drought stress, and studying its responses to drought stress may provide important information on the mechanisms underlying its drought tolerance, as well as improve its drought adaptation and that of other crops. In this study, two proso millet cultivars, NM5 and JS6, and the responses of their leaves to drought were analyzed and discussed.

\section{Overall analysis of transcriptional information of JS6 and NM5 cultivars}

From the RNA-seq data, 115,660 transcripts and 59,035 unigenes were obtained with mean lengths of 1367 and $1080 \mathrm{bp}$, respectively. Among them, 33,634 unigenes were successfully annotated in at least one of the four public databases, namely, nr, SWISS-PROT, COG, and
KEGG, using the BLASTX algorithm. These data will further enrich the transcriptome information of proso millet.

When plants experience drought stress, their gene expression levels commonly show extensive changes [47]. In the present study, in response to PEG-induced water stress, drastic transcriptional changes occurred at T6 in both cultivars and more DEGs were observed in the sensitive cultivar than in the tolerant one. More DEGs were identified in T6 treatments than in T24 treatments, compared with their respective controls in both cultivars, whereas the number of DEGs between JS6T6 and NM5T6 was found to be the lowest (Fig. 4a). These results suggested that increased transcriptional changes occurred at T6 in both cultivars, and the drought-tolerant cultivar may maintain highly stable gene expression levels during drought stress. Yates et al. [48] reported similar findings and attributed their results to the different responses in drought-sensitive and drought-tolerant plants; this characteristic may be caused by the different sensitivities of the two plants to drought.

In response to PEG-induced water stress, the sensitive cultivar exhibited more DEGs, especially up-regulated genes, which were involved in regulating responses to stimulus and biological regulation processes, than the drought-tolerant cultivar (Fig. 9b). However, more of 

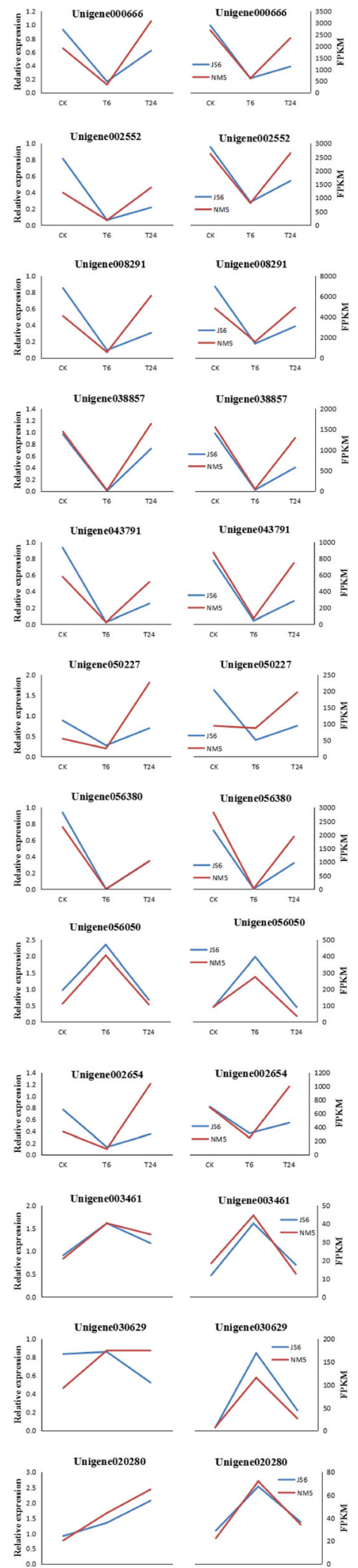

Fig. 12 Expression patterns of twelve candidate transcripts measured in JS6 and NM5 via qRT-PCR (left side) and RNA-seq (right side). CK, T6, and T24 represent different treatments using Hoagland solution containing 20\% PEG-6000 for 0, 6, and 24 h, respectively

these genes maintained higher transcript levels in the drought-tolerant cultivar, regardless of the presence or absence of simulated drought treatment (Fig. 9c). Under drought conditions, the drought-tolerant cultivar exhibited a lower requirement of intensifying the regulation of relevant genes than the sensitive cultivar. This phenomenon may be due to the less severe conditions and higher transcript levels of relevant genes in the droughttolerant cultivar compared with the sensitive cultivar.

\section{Transcriptional changes related to plant photosynthesis}

In the presence of simulated drought stress, the genes related to plant photosynthesis were most suppressed under T6 (Fig. 7a). Similar expression profiles were found in the genes related to the chlorophyll content (Fig. 7b). These findings agreed with the characteristics of plant photosynthesis in the presence of drought stress. Previous studies have demonstrated that a plant's photosynthetic activity is reduced under drought stress [33, 49,50], and photosynthesis-related genes are preferentially repressed under drought stress $[17,48]$. Notably, qRT-PCR data also presented similar characteristics. On the basis of functional annotation, Unigene 000666 was predicted to encode subunit $\mathrm{O}$ in photosystem $\mathrm{I}$, which was predicted to be related to the intrinsic component of the membrane. Unigene 008291 and Unigene 002552 putatively encode subunits II and III in the photosystem I reaction center in the chloroplast, respectively, and both were predicted to be related to cellular metabolic processes. Unigene002654 was predicted to encode a $22 \mathrm{kD}$ protein in the photosystem II reaction center in chloroplast, which was predicted to be related to energy metabolic processes. Unigene 038857, Unigene 043791, and Unigene 056380 were predicted to encode $\mathrm{CP} 26$ precursor, chlorophyll a-b binding apoprotein, and CP24 and $\mathrm{P} 4$ chlorophyll a-b binding protein, respectively, which were predicted to contribute to important metabolic and proteinmodification processes. Unigene 050227 putatively encoded the photosynthetic NDH subunit of lumenal location 2 in the chloroplast. The tested genes related to plant photosynthesis were all repressed at T6 and promoted at T24 (Fig. 12).

The genes related to plant photosynthesis were most recovered and up-regulated after drought treatment from $6 \mathrm{~h}$ to $24 \mathrm{~h}$ in both cultivars. However, the genes were most repressed under T24 in JS6 and most induced under T24 in NM5 compared with their respective 


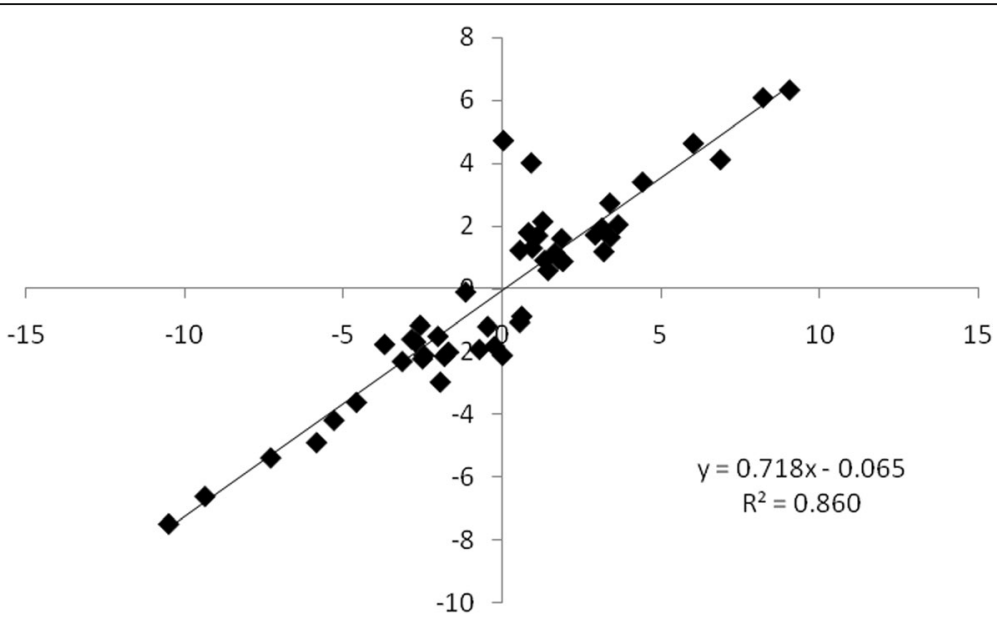

Fig. 13 Correlation between qRT-PCR and RNA-seq based on their respective data from the twelve candidate transcripts. Each point represents a fold change value of expression at T6 or T24 compared with that of CK

controls (Additional file 2: Figure S1c). The results of KEGG pathway enrichment analysis and qRT-PCR data also produced similar characteristics (Figs. 10b and 12). The photosynthesis-related genes that were repressed under drought stress could more easily return to relatively normal levels in the drought-tolerant cultivar than in the drought-sensitive cultivar. Previous studies have also demonstrated that more suppressed genes related to the photosynthetic apparatus are found in the sensitive pool than in the tolerant one [48], possibly because drought stress reduces the photosynthetic capacity of plants. Improved strategies on regulating drought tolerance in drought-tolerant cultivars, including increased expression levels of the genes related to ROS scavengers and proline accumulation, help the plants reduce ROS injury, survive drought stress, and maintain productivity. Photosynthetic recovery contributes to plant recovery after drought [51]. Thus, when stress injury is mitigated after a series of defence responses, drought-tolerant cultivars recover more easily than drought-sensitive cultivars, which maintain large portions of their photosynthesis apparatus. Drought stress would have lower adverse effects on crop growth and yield of tolerant cultivars than on those of sensitive ones.

\section{Changes in proso millet cultivars in response to oxidative stress}

Drought treatment increases MDA accumulation [52-54], and drought-sensitive cultivars accumulate more MDA than tolerant ones $[54,55]$. The present study showed that the MDA contents increased in both cultivars as the stress time continued, and the MDA content in JS6 was consistently higher than that in NM5, regardless of the presence or absence of PEG-induced water stress (Fig. 1). The results indicated that the drought-sensitive cultivar JS6 suffered higher levels of ROS injury than the droughttolerant one under PEG-induced water stress. Consistent with these results, more genes with high transcript levels that were predicted to be related to ROS scavenging enzyme in the drought-tolerant cultivar (Fig. 5b) were identified in this study. Regardless of the presence or absence of PEG-induced water stress, more genes related to proline biosynthesis maintained higher transcript levels in the tolerant cultivar than in the sensitive one (Fig. 6), thereby contributing to the low levels of ROS injury in the drought-tolerant cultivar. During osmotic stress, proline accumulation could protect proteins and membranes by protecting the enzymes of the glutathione-ascorbate cycle or enhancing their activities [2]. Thus, in response to drought stress, tolerant cultivars were more effective than sensitive cultivars in protecting the membrane system from the synergistic effects of ROS scavenging and osmotic regulation systems, regulating ROS homeostasis, reducing ROS injury, and improving drought tolerance.

In the presence of simulated drought stress, APXs and CATs showed faster transcriptional responses than PODs and SODs, and the drought-responsive genes involved in ROS scavenging systems in the droughttolerant cultivar showed delayed transcriptional responses compared with those in the sensitive cultivar. The synergistic effects and non-synchronization of the ROS scavenging system have been reported in previous studies on maize [56] and rice [57]. ROS scavenging enzyme activity in the drought-tolerant cultivars was higher than that in sensitive ones [57]. These findings are helpful to further elucidate the mechanisms of the response of proso millet to future systematic study on changes in the proline content and ROS scavengers in the presence of drought stress among different droughttolerant cultivars. 


\section{Effects of plant hormones on drought tolerance and growth of proso millet}

Compared with the drought-sensitive cultivar JS6, JAZ was suppressed in the JA signalling pathways in the tolerant cultivar NM5 under simulated drought stress. Under drought stress, the accumulation of active JA was probably higher in the drought-tolerant cultivar, which repressed JAZ and facilitated the JA signal transduction pathway, than in the sensitive cultivar. JA is important in the response to environmental stimuli [22-24]. It can promote ROS detoxification enzyme systems and ascorbic-recycling genes [26]. The abundance of ascorbate is implicated in the activation threshold of hormone signals and in hormone cross-talk [29]. JAZ can repress the expression of JA-responsive genes by suppressing the transcription factor in plant cells with low JA levels [58]. Under environmental stimuli, the accumulation of active JA stimulates JAZ degradation via the ubiquitin/ $26 \mathrm{~S}$ proteasome pathway to release the transcription factors from repression and trigger JA signalling pathways [59]. Thus, in the presence of drought, the different response strategies of the JA signal transduction pathway in the drought-tolerant cultivar may be one of the driving forces underlying drought stress tolerance. During drought stress, the high accumulation of active JA in the drought-tolerant cultivar possibly facilitated the JA signal transduction pathway, promoted ROS scavenging system, reduced ROS injury, and elevated cell membrane stability, all of which mitigated the stress conditions and made photosynthetic recovery easy.

Compared with JS6, auxin and cytokinin in the signal transduction pathways were repressed under T6 in NM5. This reaction was helpful to regulate stomatal closure in the drought-tolerant cultivar under stress treatment. Moreover, cytokinin in the signal transduction pathways was induced under T24 in NM5. This finding agreed with the hypotheses that drought-tolerant cultivars possess greater recovery capacities after a series of defence responses compared with sensitive cultivars. Furthermore, the JA and ABA signal transduction pathways were promoted under each stress treatment in NM5, which helped regulate stomatal closure and activate downstream defence responses during stress treatments. A further systematic study on the changes in the plant hormones of different drought-tolerant cultivars during response to drought will provide important information to further elucidate the mechanisms of the response of proso millet.

\section{Conclusion}

In the presence of simulated drought stress, NM5 maintained highly stable gene expression levels and carried more genes with high transcript levels that were predicted to be related to the ROS scavenging enzyme compared with JS6. Compared with drought-sensitive cultivars, the different regulation strategies of the JA signal transduction pathway in drought-tolerant cultivars may be one of the driving forces of drought stress tolerance.

\section{Methods \\ Plant materials and drought treatment}

In this study, PEG-induced osmotic stress was used to simulate drought stress. On the basis of germination rates, drought-sensitive and drought-tolerant materials for subsequent analysis were selected among 37 core Chinese proso millet cultivars. All the above-mentioned proso millet cultivars have been released in China and all the test materials were provided by the Minor Grain Research Group of the College of Agronomy, Northwest A \& F University from cultivated plants in the fields.

A total of 600 seeds of similar sizes and appearance were selected from each proso millet cultivar and randomly divided into six groups for germination. All seeds were pre-processed via oven drying at $30{ }^{\circ} \mathrm{C}-35^{\circ} \mathrm{C}$ for 48-72 h. Subsequently, 100 seeds from each cultivar were distributed in a plastic box $(12 \mathrm{~cm} \times 12 \mathrm{~cm} \times 8 \mathrm{~cm})$ with filter paper and treated with $8 \mathrm{~mL}$ of distilled water as the control and $8 \mathrm{~mL}$ of $20 \%$ PEG 6000 solution as the intervention. Finally, seeds were grown in a covered plastic box under a photoperiod of $0 \mathrm{~h}$ light $/ 24 \mathrm{~h}$ dark and humidity of $70 \%$ at $25^{\circ} \mathrm{C}$ for 3 days. The germination rates were then measured. The cultivars in the control group with germination rates higher than $95 \%$ and those in the treatment group with the highest or lowest relative germination rates were selected as the drought-tolerant and drought-sensitive cultivars, respectively, for subsequent analysis.

Seeds of both cultivars were germinated and grown in distilled water in plastic boxes for 3 days under a photoperiod of $0 \mathrm{~h}$ light $/ 24 \mathrm{~h}$ dark and humidity of $70 \%$ at $25^{\circ} \mathrm{C}$. The plants were cultivated in Hoagland solution under a photoperiod of $16 \mathrm{~h}$ light $/ 8 \mathrm{~h}$ dark and humidity of $70 \%$ at $25^{\circ} \mathrm{C}$. At the three-leaf stage, the seedlings of both cultivars were treated for 0 (CK), 6 (T6), and $24 \mathrm{~h}$ (T24) with Hoagland solution containing 20\% PEG6000. After the treatments, leaf tissues from each group were sampled for subsequent analysis, rapidly frozen in liquid nitrogen, and stored at $-80^{\circ} \mathrm{C}$.

\section{Measurements of MDA, RNA extraction, transcriptome sequencing, and de novo sequence assembly}

MDA levels were measured as described previously [60]. Total RNA from the collected leaf tissues was extracted using TRIzol ${ }^{\varpi}$ reagent (Invitrogen) in accordance with the manufacturer's instructions. The integrity of the extracted RNAs was assessed via agarose gel electrophoresis using Agilent 2100 Bioanalyzer (AgilentTechnologies, Palo Alto, 
CA, USA). The quantity was determined with a NanoDrop 8000 spectrophotometer (NanoDrop, Wilmington, DE, USA). The quality of all the extracted RNAs was accepted for library construction containing total RNA with RIN values $>6.3$ and $28 \mathrm{~S}: 18 \mathrm{~S}$ ratios $\geq 1.1$.

Total mRNAs were enriched by using oligo (dT) magnetic beads and further sheared into short fragments by adding a fragmentation buffer. These RNA fragments were involved in the synthesis of first-strand cDNA with random hexamers, followed by the synthesis of secondstrand cDNA with DNA polymerase I and RNase $\mathrm{H}$ (Invitrogen). After purification using a QiaQuick PCR extraction kit (QIAGEN), end-repaired and A-tailed sequencing adaptors were ligated onto the cDNA fragments. Suitable fragments were purified using AMPure $\mathrm{XP}$ beads and amplified via PCR amplification to construct a library for subsequent sequencing. Transcriptome sequencing was performed on the $\mathrm{HiSeq}^{\mathrm{mm}} 4000$ platform (SageneBiotech Co. Ltd., Guangzhou, China). The raw image data from sequencing were transformed into raw reads via base calling. High-quality clean reads were obtained by strict filtering steps, including removal of adapter sequences, reads containing more than $10 \%$ unknown bases, and low-quality reads. The obtained high-quality clean reads were mixed and assembled using Trinity program.

\section{Functional annotation and DEG analysis}

To determine the putative function, we searched the assembled unigenes against the databases, including the $\mathrm{nr}$ (http://www.ncbi.nlm.nih.gov/), SWISS-PROT (http:// www.expasy.ch/sprot/), KEGG (http://www.genome.jp/ kegg/), and KOG (http://www.ncbi.nlm.nih.gov/cog/) databases by using the BLASTX algorithm with an $e$ value of $<1 \mathrm{e}^{-5}$. The assembled unigenes were annotated with the GO database by the Blast2GO program based on NR annotation, and GO functional classification of unigenes was performed using WEGO.

The expression values of reads were normalized with Reads Per Kilobase of exon Model per Million mapped reads. The threshold $P$ was adjusted using the false discovery rate (FDR) in multiple hypothesis testing. In this study, the cut-off was set as FDR $\leq$ 0.05 and $|\log 2 \mathrm{FC}| \geq 1$ to determine the DEGs using edgeR package (http://www.r-project.org/). All the DEGs were subjected to enrichment analysis of GO functions and KEGG pathways.

\section{Quantitative real-time PCR analysis}

Additional file 1: Table S1 shows the sequence of the primers involved in the qRT-PCR experiments. SYBR premix Ex Taq kit (TaKaRa, Dalian, China) was used in the experiment, and qRT-PCR was performed on an ABI 7500 Real-Time System (Applied Biosystems) with first- strand cDNA as the template. To confirm the DEG results, 12 transcripts were randomly selected for qRTPCR validation. Actin served as the internal control. The fold change in the expression levels of target genes was calculated via relative quantification $\left(2^{-\triangle \Delta C T}\right)$ [13].

\section{Additional files}

\begin{abstract}
Additional file 1: Table S1. Information on the primers involved in the qRT-PCR experiments. Table S2. Germination rates of 37 core Chinese proso millet cultivars. Table S3. Information on the pathways with the most quantities of DEGs in different KEGG classes. Table S4. Information on the top $30 \mathrm{GO}$ categories with the most quantities of DEGs in the three major GO classes in both cultivars. Table S5. Information on the DEGs involved in the KEGG pathways in both cultivars. Table S6. Level 3 GO annotations of the DEGs in three major GO classes in both cultivars. Table S7. Profiles of the top 10 pathways with the most quantities of DEGs and photosynthesis pathway based on KEGG pathway enrichment analysis. (XLSX $36 \mathrm{~kb}$ )

Additional file 2: JS6 and NM5 were treated with 20\% PEG-6000 solution for 6 (JS6T6 and NM5T6, respectively) and 24 h (JS6T24 and NM5T24, respectively) or with water (control group; JS6CK and NM5CK, respectively). Figure S1. DEGs involved in photosynthesis in JS6 and NM5 in the presence and absence of drought treatments. a Euler diagram of the DEGs involved in photosynthesis from comparisons between the control and T6 treatment groups, including up- and down-regulated genes in JS6T6 and NM5T6. b Euler diagram of the DEGs involved in photosynthesis from comparisons between the T6 and T24 treatment groups, including up- and downregulated genes. c Euler diagram of the DEGs involved in photosynthesis from comparisons between the control and T24 treatment groups, including up- and down-regulated genes in JS6T24 and NM5T24. Figure S2. Chlorophyll content-related genes in JS6 and NM5 differentially expressed in the presence and absence of drought treatments. a Euler diagram of the DEGs related to chlorophyll from comparisons between the control and T6 treatment groups, including up- and down-regulated genes in JS6T6 and NM5T6. b Euler diagram of the DEGs related to chlorophyll from comparisons between T6 and T24 treatment groups, including up- and down-regulated genes. c Euler diagram of the DEGs related to chlorophyll from comparisons between the control and T24 treatment groups, including up- and down-regulated genes in JS6T24 and NM5T24. (ZIP $544 \mathrm{~kb})$
\end{abstract}

\begin{abstract}
Abbreviations
ABA: Abscisic acid; BLAST: Basic local alignment search tool; CAT: Catalase; cDNA: Complementary deoxyribonucleic acid; DEGs: Differentially expressed genes; FC: Fold change; FDR: False discovery rate; FPKM: Fragments per kilobase of exon per million fragments mapped; GO: Gene Ontology; JA: Jasmonic acid; JAZ: Jasmonate ZIM domain proteins; MDA: Malondialdehyde; POD: Peroxidase; qRT-PCR: Quantitative real-time polymerase chain reaction; RNA-seq: RNA sequencing; ROS: Reactive oxygen species; SOD: Superoxide dismutase
\end{abstract}

\section{Acknowledgements}

The authors would like to thank SageneBiotech Co. Ltd. (Guangzhou, China) for assistance with raw data processing and bioinformatics analyses. We also thank Biomarker Co. Ltd. (Beijing, China) for suggestions on bioinformatics analyses.

\section{Authors' contributions}

$Z Y Y, G X L, W P K$, and FBL designed the experiments. ZYY, LJ, and GXW conducted the experiments. ZYY, GXL, and GJF analyzed the data. ZYY, $\mathrm{GXL}$, and YP wrote the paper. All the authors read and approved the final manuscript. 


\section{Funding}

The study was mainly sponsored by the funds from National Millet Crops Research and Development System (CARS-06-13.5-A26), National Natural Science Foundation of China (31371529), National Science and Technology Supporting Plan (2014BAD07B03) and Program for the Key Science and Technology in Shaanxi Province (S2018-YF-TSLNY-0005) for collection, analysis and processing of data, and was partially supported by Minor Grain Crops Research and Development System of Shaanxi Province (2009-2018) for editing the manuscript.

\section{Availability of data and materials}

All the raw reads of RNA sequencing data were deposited in the NCBI Short Read Archive (SRA) database under the accession number SRP144636 (https://www.ncbi.nlm.nih.gov/sra/SRP144636), as associated with the BioProject PRJNA454008. The transcriptome shotgun assembly data were deposited at DDBJ/EMBL/GenBank under the accession number GHHA01000000.

\section{Ethics approval and consent to participate}

Not applicable.

\section{Consent for publication}

Not applicable.

\section{Competing interests}

The authors declare that they have no competing interests.

\section{Received: 22 May 2018 Accepted: 30 August 2019}

\section{Published online: 11 September 2019}

\section{References}

1. Boyer JS. Plant productivity and environment. Science. 1982;218:443-8.

2. Khan MS, Ahmad D, Khan MA. Utilization of genes encoding osmoprotectants in transgenic plants for enhanced abiotic stress tolerance. Electron J Biotechnol. 2015;18:257-66.

3. Sarwata M, Tuteja N. Hormonal signaling to control stomatal movement during drought stress. Plant Gene. 2017;11:143-53.

4. Chaves MM, Oliveira MM. Mechanisms underlying plant resilience to water deficits: prospects for water-saving agriculture. J Exp Bot. 2004;55:2365-84.

5. Foley JA, Ramankutty N, Brauman KA, Cassidy ES, Gerber JS, Johnston M, et al. Solutions for a cultivated planet. Nature. 2011;478:337-42.

6. Qin DH, Ding YH, Wang SW, Wang SM, Lin ED, et al. Ecological and environmental change in West China and its response strategy. Adv Earth Science. 2002;17:314-9 (in Chinese).

7. Zhao H, Wang RY, Ma BL, Xiong YC, Qiang SC, Wang CL, et al. Ridge-furrow with full plastic film mulching improves water use efficiency and tuber yields of potato in a semiarid rainfed ecosystem. Field Crop Res. 2014;161:137-48.

8. Dong J, Wang H, Chen L, Wang J, Qiao Z. Analysis on genetic diversity of 96 proso millet germplasms. J Hebei Agric Sci. 2014;18:1-4 (in Chinese).

9. Qu Y, Su W, Zhang PP, Li C, Gao JF, Gao XL, et al. Effects of different water harvesting on soil water, growth and yield of the proso millet (Panicum miliaceum L.) in a semiarid region of Northwest China. J Agric Sci. 2012:4:106-13 (in Chinese).

10. Su W, Zhang YP, Qu Y, Li C, Miao JY, Gao XL, et al. Effects of mulching patterns on soil water, broomcorn millet growth, photosynthetic characteristics and yield in the dryland of loess plateau in China. Chin J Appl Ecol. 2014;25:3215-22 (in Chinese).

11. Hunt HV, Denyer K, Packman LC, Jones MK, Howe CJ. Molecular basis of the waxy endosperm starch phenotype in broomcorn millet (Panicum miliaceum L.). Mol Biol Evol. 2010;7:1478-94.

12. Wang RY, Hunt HV, Qiao ZJ, Wang L, Han YH. Diversity and cultivation of broomcorn millet (Panicum miliaceum L.) in China: a review. Econ. Bot 2016;70:332-42

13. Yue $H$, Wang L, Liu H, Yue WJ, Du XH, Song WN, et al. De novo assembly and characterization of the transcriptome of broomcorn millet (Panicum miliaceum L.) for gene discovery and marker development. Front. Plant Sci. 2016;7:1083

14. Hu Y, Zhu J, Liu F, Zhang Z, Chai Y, Song WN. Genetic diversity among Chinese landraces and cultivars of broomcorn millet (Panicum miliaceum) revealed by the polymerase chain reaction. Ann Appl Biol. 2008;153:357-64.
15. Zhang YY, Han HK, Zhang DZ, Li J, Gong XW, Feng BL, et al. Effects of ridging and mulching combined practices on proso millet growth and yield in semi-arid regions of China. Field Crop Res. 2017;213:65-74.

16. Price $\mathrm{AH}$, Cairns JE, Horton $\mathrm{P}$, Jones $\mathrm{HG}$, Griffiths $\mathrm{H}$. Linking drought resistance mechanisms to drought avoidance in upland rice using a QTL approach: progress and new opportunities to integrate stomatal and mesophyll responses. J Exp Bot. 2002;53:989-1004.

17. Chen W, Yao Q, Patil GB, Agarwal G, Deshmukh RK, Lin L, et al. Identification and comparative analysis of differential gene expression in soybean leaf tissue under drought and flooding stress revealed by RNA-Seq. Front Plant Sci. 2016;7:1044.

18. Yamaguchi-Shinozaki K, Shinozaki K. Transcriptional regulatory networks in cellular responses and toletance to dehydration and cold stresses. Annu Rev Plant Biol. 2006;57:781-803

19. Wilkinson S, Davies WJ. Drought, ozone, ABA and ethylene: new insights from cell to plant to community. Plant Cell Environ. 2010;33:510-25.

20. Peleg Z, Blumwald E. Hormone balance and abiotic stress tolerance in crop plants. Curr Opin Plant Biol. 2011;14:290-5.

21. Acharya B, Assmann S. Hormone interactions in stomatal function. Plant Mol Biol. 2009;69:451-62

22. Devoto A, Turner JG. Jasmonate-regulated Arabidopsis stress signaling network. Physiol Plant. 2005;123:161-72.

23. Giri AP, Wünsche $H$, Mitra S, Zavala JA, Muck A, Svatos A, Baldwin IT. Molecular interactions between the specialist herbivore Manduca sextta (Lepidoptera, Sphingidae) and its natural host Nicotiana attenuata. VII. Changes in the plant's proteome. Plant Physiol. 2006;142:1621-41.

24. Yan Y, Stolz S, Chételat A, Reymond P, Pagni M, Dubugnon L, Farmer EE. A downstream mediator in the growth repression limb of the jasmonate pathway. Plant Cell. 2007;19:2470-83.

25. Noctor G, Foyer C. Ascorbate and glutathione: keeping active oxygen under control. Annu Rev Plant Physiol Plant Mol Biol. 1998:49:249-79.

26. Nafie E, Hathout T, Mokadem ASA. Jasmonic acid elicits oxidative defense and detoxification systems in Cucumis melo L. cells. Braz J Plant Physiol. 2011;23:161-74.

27. Jimenez A, Hernandez JA, Del RL, Sevilla F. Evidence for the presence of the ascorbate-glutathione cycle in mitochondria and peroxisomes of pea leaves. Plant Physiol. 1997;114:275-84.

28. Asada K. The water-water cycle in chloroplasts: scavenging of active oxygen and dissipation of excess photons. Annu Rev Plant Physiol Plant Mol Biol. 1999;50:601-39.

29. Bartoli CG, Casalongué CA, Simontacchi M, Marquez-Garcia B, Foyer CH. Interactions between hormone and redox signalling pathways in the control of growth and cross tolerance to stress. Environ Exp Bot. 2013;94:73-88.

30. Pignocchi C, Fletcher J, Barnes J, Foyer $\mathrm{CH}$. The function of ascorbate oxidase $(\mathrm{AO})$ in tobacco (Nicotiana tabacum L.). Plant Physiol. 2003;132:1631-41.

31. Davey MW, Stals E, Panis B, Keulemans J, Swennen RL. High-throughput determination of malondialdehyde in plant tissues. Anal Biochem. 2005;347:201-7.

32. Valliyodan $\mathrm{B}$, Nguyen $\mathrm{HT}$. Understanding regulatory networks and engineering for enhanced drought tolerance in plants. Curr Opin Plant Biol. 2006;9:189-95.

33. Chen W, He S, Liu D, Patil GB, Zhai H, Wang F, et al. A sweetpotato geranylgeranyl pyrophosphate synthase gene, IbGGPS, increases carotenoid content and enhances osmotic stress tolerance in Arabidopsis thaliana. PLoS One. 2015;16:e0137623.

34. Knight $H$, Knight MR. Abiotic stress signaling pathways: specificity and cross-talk. Trends Plant Sci. 2001:6:262-7.

35. Mittler R. Oxidative stress, antioxidants, and stress tolerance. Trends Plant Sci. 2002;7:405-10.

36. Diplock AT. Defence against reactive oxygen species. Free Radic Res. 1998 29:463-7.

37. Alscher RG, Donahue JL, Cramer CL. Reactive oxygen species and antioxidants: relationships in green cells. Physiol Plant. 1997;100:224-33.

38. Liu Y, Pan QH, Yang HR, Liu YY, Huang WD. Relationship between $\mathrm{H}_{2} \mathrm{O}_{2}$ and jasmonic acid in pea leaf wounding response. Russ J Plant Physiol. 2008:55:851-62.

39. Lamb C, Dixon RA. The oxidative burst in plant disease resistance. Annu. Rev. plant Physiol. Plant Mol. Biol. 1997;48:251-75. 
40. Bohnert HJ, Sheveleva E. Plant stress adaptations - making metabolism move. Curr Opin Plant Biol. 1998;1:267-74.

41. Blumwald E. Sodium transport and salt tolerance in plants. Curr Opin Cell Biol. 2000;12:431-4.

42. Clark MB, Mercer TR, Bussotti G, Leonardi T, Haynes KR, Crawford J, et al. Quantitative gene profiling of long noncoding RNAs with targeted RNA sequencing. Nat Methods. 2015;12:339-42.

43. Xu K, Sun F, Chai G, Wang Y, Shi L, Liu S, Xi Y. De Novo assembly and transcriptome analysis of two contrary tillering mutants to learn the mechanisms of tillers outgrowth in switchgrass (Panicum virgatum L.). front. Plant Sci. 2015;6:749.

44. Zhan J, Thakare D, Ma C, Lloyd A, Nixon NM, Arakaki AM, et al. RNA sequencing of laser-capture microdissected compartments of the maize kernel identifies regulatory modules associated with endosperm cell differentiation. Plant Cell. 2015;27:513-31.

45. Wang RY, Wang HG, Liu XY, Lian S, Chen L, Qiao ZJ, Mclnerney CE, Wang L. Drought-induced transcription of resistant and sensitive common millet varieties. J Animal Plant Sci. 2017;27(4):1303-14.

46. Lei Y, Xu Y, Hettenhausen C, Lu CK, Shen GJ, Zhang CP, et al. Comparative analysis of alfalfa (Medicago sativa L.) leaf transcriptomes reveals genotypespecific salt tolerance mechanisms. BMC Plant Biol. 2018;18:35.

47. Long Y, Zhang JW, Tian XJ, Wu SS, Zhang Q, Zhang JP, et al. De novo assembly of the desert tree Haloxylon ammodendron (C. A. Mey.) based on RNA-Seq data provides insight into drought response, gene discovery and marker identification. BMC Genomics. 2014;15:1111.

48. Yates SA, Swain MT, Hegarty MJ, Chernukin I, Lowe M, Allison GG, et al. De novo assembly of red clover transcriptome based on RNA-Seq data provides insight into drought response, gene discovery and marker identification. BMC Genomics. 2014;15:453

49. Tabaeizadeh Z. Drought-induced responses in plant cells. Int Rev Cytol. 1998;182:193-247.

50. Krasensky J, Jonak C. Drought, salt, and temperature stress-induced metabolic rearrangements and regulatory networks. J Exp Bot. 2012;63:1593-608.

51. Chaves MM, Flexas J, Pinheiro C. Photosynthesis under drought and salt stress: regulation mechanisms from whole plant to cell. Ann Bot. 2009;103:551-60.

52. Fayez KA, Bazaid SA. Improving drought and salinity tolerance in barley by application of salicylic acid and potassium nitrate. J Saudi Soc Agric Sci. 2014;13:45-55

53. Zhang PP, Feng BL, Wang PK, Gao XL, Ta JM, Chai Y, Song H. Leaf senescence and protective enzyme system of broomcorn millet under drought condition. Agric Res Arid Areas. 2010;28:99-8 (in Chinese).

54. Zhang MS, Tan F, Xie B, Zhang QT, Fu YF, Yang CX, Yang YH. Relationship of peroxidation of membrane-lipid and membrane protection system in Sweetpotato under water stress with drought resistance. Sci Agric Sin. 2003;36:1395-8 (in Chinese).

55. Sun FG, Liu XS. Study on drought-resistance identification indices of crops. J Anhui Agric Sci. 2009;37:12494-5 (in Chinese).

56. Wang ZW, Mou SW, Yan LL, Han QF, Yang BP. Effects of physiological and biochemical characteristics and growth under water stress in seedling of spring maize. Acta bot Boreal-occident Sin. 2013;33:343-51 (in Chinese).

57. Zhao HW, Wang XP, Yu MF, Sha HJ, Jia Y, Yu TC, Zou DT. Effect of drought stress and rewatering on antioxidant system and proline in rice during tillering stage. J Northeast Agric Univ. 2016;47:1-7 (in Chinese).

58. Thines B, Katsir L, Melotto M, Niu Y, Mandaokar A, Liu G, et al. JAZ repressor proteins are targets of the SCFCOI1 complex during jasmonate signalling. Nature. 2007:448:661-5.

59. Chung HS, Howe GA. A critical role for the TIFY motif in repression of jasmonate signaling by a stabilized splice variant of the JASMONATE ZIMdomain protein JAZ10 in Arabidopsis. Plant Cell. 2009;21:131-45.

60. Heath RL, Packer L. Photoperoxidation in isolated chloroplasts. I. Kinetics and stoichiometry of fatty acid peroxidation. Arch Biochem Biophys. 1968;125:189-98.

\section{Publisher's Note}

Springer Nature remains neutral with regard to jurisdictional claims in published maps and institutional affiliations.

\section{Ready to submit your research? Choose BMC and benefit from}

- fast, convenient online submission

- thorough peer review by experienced researchers in your field

- rapid publication on acceptance

- support for research data, including large and complex data types

- gold Open Access which fosters wider collaboration and increased citations

- maximum visibility for your research: over $100 \mathrm{M}$ website views per year

At BMC, research is always in progress.

Learn more biomedcentral.com/submissions 